\title{
Consecuencias del aprendizaje organizativo en las relaciones comerciales entre empresas
}

José A. López Sánchez jangel@unex.es Avenida de Elvas s/n, 06071 , Badajoz-España

Doctor en Ciencias Económicas y Empresariales (Universidad de Oviedo). Doctor en la Universidad de Extremadura, Facultad de Ciencias Económicas y

Empresariales, Departamento de Dirección de Empresas y Sociología.

María Leticia Santos Vijande Isantos@uniovi.es

Avenida del Cristo s/n, 33071 Oviedo-España Doctora en Ciencias Económicas y Empresariales Profesora Titular de Universidad. Subdirectora del IUDE Departamento de

Administración de Empresas Facultad de Economía y Empresa.

Juan A. Trespalacios Gutiérrez jtrespa@uniovi.es Avenida del Cristo s/n, 33071 Oviedo-España

Catedrático de Universidad y Director del Instituto Universitario de la Empresa (IUDE) en la Universidad de Oviedo, Facultad de Ciencias Económicas y Empresariales.

Artículo Tipo: de investigación Según Clasificación Colciencias

Fecha de recepción: 22 de Diciembre de 2010

Fecha de corrección: 22 de junio de 2011 Fecha de aprobación: 22 de junio de 2011
Consequences of organizational learning in commercial business relationships

Conséquences de l'apprentissage organisationnel dans les relations commerciales entre entreprises

\section{Resumen}

El objetivo principal de este estudio es analizar la influencia del aprendizaje organizativo (AO) sobre la satisfacción y la lealtad en las relaciones comerciales entre empresas. Para ello se plantea un marco teórico donde se adopta como unidad de análisis la relación diádica que mantienen las empresas fabricantes con su principal distribuidor. En el análisis de los datos se emplean sistemas de ecuaciones estructurales con una muestra de empresas españolas. Los resultados empíricos obtenidos aportan evidencias acerca de que el $A O$ del fabricante es un antecedente de los resultados alcanzados en los intercambios relacionales. Así, el mayor grado de AO de la empresa fabricante incide de modo directo en el grado de satisfacción del distribuidor principal y también actúa de modo indirecto sobre su grado de lealtad. Se corrobora además que dicho AO potencia de un modo directo los resultados empresariales del fabricante. Las limitaciones del estudio son las típicas asociadas al uso de la investigación basada en encuesta. Por último, la varianza del método común no fue un problema en este estudio.

Palabras clave: aprendizaje organizativo, satisfacción, lealtad, canales de distribución, relaciones comerciales. 


\section{Consequences of organizational learning in commercial business relationships}

\author{
Consecuencias del aprendizaje \\ organizativo en las relaciones \\ comerciales entre empresas
}

\author{
Conséquences de l'apprentissage \\ organisationnel dans les relations \\ commerciales entre entreprises
}

\section{Conséquences de l'apprentissage organisationnel dans les relations commerciales entre entreprises}

\author{
Consecuencias del aprendizaje \\ organizativo en las relaciones \\ comerciales entre empresas
}

Consequences of organizational learning in commercial business relationships

\section{Résumée}

L'objectif principal de cette étude est celui d'analyser l'influence de l'apprentissage organisationnel (AO) sur la satisfaction et la loyauté dans les relations commerciales entre entreprises. Pour cela, on propose un cadre théorique où l'on adopte comme unité d'analyse la relation dyadique des entreprises productrices avec leur principal distributeur. Pour l'analyse des données on a utilisé des systèmes d'équations structurelles en prenant comme échantillon des entreprises espagnoles. Les résultats empiriques obtenus apportent comme évidence que l'AO du producteur constitue un précédent des résultats atteints dans les échanges relationnels. Ainsi, le plus haut degré d'AO de l'entreprise productrice a une incidence directe sur le degré de satisfaction du distributeur principal et agit aussi de façon indirecte sur le degré de loyauté. On peut corroborer en plus que cet $A O$ potentialise d'une façon directe les résultats d'affaires du producteur. Les limitations de l'étude sont typiques, celles associées à l'usage de la recherche basée sur des enquêtes. Finalement, la variance de la méthode commune n'a pas été un problème pour cette étude.

Mots clef: rapprentissage organisationnel, satisfaction, loyauté, canaux de distribution, relations commerciales. 


\section{Consecuencias del aprendizaje organizativo en las relaciones comerciales entre empresas}

\section{Introducción}

El aprendizaje organizativo (AO) es ampliamente reconocido en la literatura como un recurso estratégico que permite mejorar la competitividad de las organizaciones (Argyris, 1991; Bell et al., 2010). Los beneficios del aprendizaje se han confirmado en multitud de aspectos de gestión como, por ejemplo, la calidad del servicio (Tucker et al., 2007), el desarrollo de nuevos productos (Akgun et al., 2006) y la orientación al mercado (Santos et al., 2005). Estas evidencias ponen de manifiesto la importancia del AO en la creación de nuevos espacios de mercado y el papel clave que ostenta en la supervivencia de las empresas ante un entorno de constante cambio.

En este contexto, el objetivo principal de este estudio es alcanzar un mayor grado de conocimiento acerca de la influencia del AO en la satisfacción y la lealtad de los socios comerciales en el ámbito de los mercados industriales. Aunque la literatura existente acerca de las relaciones comprador-vendedor identifica muchas variables relacionales de resultado, en esta investigación la atención se centra en la satisfacción y la lealtad, dado que estas variables constituyen poderosos mecanismos para garantizar un rendimiento a largo plazo en las relaciones empresariales (Ittner y Larcker, 2003). Las empresas que trabajan bajo esta premisa buscan la excelencia a través del desarrollo de relaciones con los clientes donde la satisfacción y la lealtad crecen con el paso del tiempo. Para estas empresas no es suficiente con prestar sólo atención a medidas de carácter financiero, como pueden ser las ventas y los beneficios, ya que dichos indicadores pueden ser considerados como una instantánea del presente que pueden dar una imagen incompleta de las relaciones con el cliente en el futuro (Narayandas, 2005).

Ahora bien, a pesar de que en el plano teórico se ha argumentado el posible efecto positivo del AO sobre la satisfacción y la lealtad del cliente, el número de investigaciones que hasta la fecha se ha preocupado por esta cuestión se puede calificar de reducido (Lapre y Tsikriktsis, 2006). Todo ello resulta sorprendente cuando ampliar nuestra comprensión acerca de esta cuestión es relevante para académicos y directivos.

Así, nos podemos plantear, ¿por qué las empresas promueven o ponen freno al desarrollo de la satisfacción y la lealtad del cliente? Una forma de abordar este interrogante es examinando los factores controlables de las compañías que pueden actuar como antecedentes de la satisfacción y la lealtad del cliente. Examinar empíricamente el efecto de estos factores puede ayudar a las empresas a gestionarlos de un modo eficaz con el propósito último de mejorar la calidad de sus relaciones comerciales. Este vacío de investigación ha propiciado que investigadores como Edmonson (2008) y Tax y Brown (1998) hayan insistido en la necesidad de desarrollar estudios empíricos al respecto. El presente trabajo también contribuye a la literatura al examinar el efecto combinado que ejercen el $\mathrm{AO}$ junto con las variables relacionales de resultado (satisfacción y lealtad) sobre los resultados empresariales. El análisis de estas relaciones causales es relevante: primero, porque proporciona una mejor comprensión acerca del papel desempeñado por el AO, la satisfacción y la lealtad en los resultados empresariales; y segundo, porque estas variables tienen una influencia importante en el desarrollo y mantenimiento de relaciones exitosas con una orientación de largo plazo. La estructura del trabajo es la siguiente: primero, se propone un modelo conceptual en el que se conectan el AO, la satisfacción, la lealtad y los resultados empresariales. La sección empírica presenta los resultados de la investigación obtenidos a partir de una muestra de 181 empresas fabricantes ubicadas en España. Por último, se presentan tanto las conclusiones como las implicaciones empresariales, y se describen las líneas de investigación futuras.

\section{Marco teórico e hipótesis}

En esta investigación se adopta el enfoque basado en los recursos (EBR) para desarrollar un marco teórico con el que se pretende conseguir un mayor grado de conocimiento acerca de cómo se pueden mejorar la satisfacción y la lealtad en los mercados industriales (Palmatier et al., 2007; Wernerfelt, 1984). Desde esta óptica el AO se 
considera un recurso estratégico para el éxito a largo plazo de una relación (Santos et al., 2005). Precisamente, se entiende que el AO ejerce un efecto positivo en la satisfacción y la lealtad. Un socio comercial que tenga por tanto un alto nivel de $A O$ se espera que ofrezca productos que se ajustan de forma precisa a las necesidades de su contraparte en la díada.

Así mismo, entendemos que una organización aprende "si a través del procesamiento de la información cambia el alcance de sus comportamientos potenciales" (Huber, 1991, p. 89). Esta aproximación coincide con la escuela de proceso del AO, la cual considera que el aprendizaje colectivo está enraizado en las capacidades cognitivas y de comportamiento de las personas que forman parte de la organización (Bell et al., 2002). La escuela de proceso del AO mantiene que los procesos de aprendizaje individual se pueden replicar a un nivel superior hasta el punto de que, al igual que los individuos, las organizaciones también son capaces de aprender cuando así lo requieren. Conforme a lo apuntado en esta investigación se adopta un constructo multidimensional del $\mathrm{AO}$, el cual, de forma sintética, se explica a continuación (López et al., 2010).

a) Adquisición de información. La información puede tener su origen en fuentes internas y externas (Argyris y Schön, 1978; Huber, 1991; Slater y Narver, 1995). La información se desarrolla internamente a través del aprendizaje congénito, el cual proviene de los fundadores de la compañía, la experiencia previa, o el aprendizaje indirecto entendido éste último como el análisis espontáneo de las acciones desarrolladas por los competidores. En otras ocasiones las empresas buscan información externa, para identificar tendencias clave, resolver problemas específicos, y comparar sus resultados con los de la competencia. Buscar información fuera de la empresa también incluye el injerto, esto es, la adquisición de organizaciones, la creación de joint ventures, o la incorporación de nuevos empleados de otras entidades.

b) Distribución de información. Este proceso tiene lugar a través de interacciones formales (por ejemplo, reuniones inter-departamentales, discusiones acerca de las necesidades futuras de la organización, y la formación cruzada) e informales entre los distintos sujetos de la organización (Schein, 1993). La comunicación en esta etapa del AO se favore- ce mediante la creación de bases de datos y redes formales, las cuales garantizan la rápida y precisa difusión de la información. Estas iniciativas se complementan además con mecanismos informales de intercambio de información, de tal forma que el conocimiento tácito adquirido por los empleados puede ser transformado en conocimiento explícito para la organización (Bontis et al., 2002).

c) Interpretación de información. La tercera etapa pretende analizar la información desde un punto de vista global (Tippins y Sohi, 2003). Por esta razón alcanzar un consenso con respecto al significado de la información y sus implicaciones es una prioridad para la organización. Con este propósito las empresas desarrollan modelos mentales compartidos y favorecen el ajuste continuo entre sus diversas áreas funcionales para un desarrollo de sus operaciones (Argyris y Schön, 1996). La interpretación compartida se ve favorecida además por la riqueza de las distintas herramientas de comunicación empleadas (Treviño et al., 2000). Sin embargo, con el propósito de interpretar la información de la manera más correcta posible las compañías, a veces, se ven en la necesidad de emprender procesos de desaprendizaje. Deben cuestionarse por tanto los modelos mentales compartidos predominantes y el conocimiento acumulado, a la vez que se deben rechazar aquellas creencias, datos, e información obsoleta que puede inducir a una toma de decisiones ineficaz (Argyris, 1996).

d) Memoria organizativa. La idea del aprendizaje colectivo ha impulsado otra dimensión del AO, a saber, la memoria organizativa. Este constructo representa todo el conocimiento acumulado por la organización. Tiene que ser almacenado adecuadamente y estar a disposición de los empleados, de tal forma que pueda ser recuperado cuando resulte necesario, lo que previene que el conocimiento se pierda a través de las rotaciones del personal. En este estudio la atención se dirige a la memoria activa, la memoria que existe en los individuos y las redes sociales (Cross y Baird, 2000; Cross et al., 2005), en vez de a la memoria pasiva, la cual está basada en las tecnologías de la información aplicada a los ordenadores. La razón es que la memoria activa es la que en última instancia determina cómo se deben conseguir los objetivos organizativos. 


\subsection{Aprendizaje organizativo y satisfacción}

La satisfacción en las relaciones comprador-vendedor se ha convertido en un imperativo estratégico para la mayoría de las empresas. Dicho estado afectivo positivo se puede materializar en "una moral incrementada, una cooperación entre los miembros del canal, y menores litigios, entre otros resultados" (Ganesan, 1994, p. 5). Los beneficios tangibles de una relación en la que existe satisfacción son mayores que los que se pueden obtener cuando no está presente, ya que la satisfacción incrementa la moral entre los miembros del canal. También resulta más probable que los litigios se vean reducidos. En este trabajo la satisfacción se define como "un estado afectivo positivo que resulta de la valoración de todos los aspectos de la relación comercial que mantiene una empresa con otra"(Anderson y Narus, 1984, p. 66).

En este contexto, la literatura revela que las empresas capaces de aprender rápidamente a modificar sus productos y formas de hacer negocios obtienen resultados favorables en la satisfacción del cliente (Hult, 1998). El AO permite anticipar cambios en las necesidades expresadas y latentes, y favorece la existencia de una cartera de productos que se ajusta a las demandas del mercado. Las empresas fabricantes que operen como una organización de aprendizaje serán capaces de conocer y anticipar los gustos, necesidades, y deseos de los distribuidores, lo que les permitirá materializar este conocimiento en una oferta de mercado lo suficientemente atractiva, debido a la flexibilidad inherente que confiere el AO para afrontar las condiciones cambiantes del mercado (Morgan y Turnell, 2003). El AO también aporta introspección acerca de por qué se sigue un determinado comportamiento cuando se trata de dar solución a los problemas planteados por los distribuidores. Se mejora por tanto la capacidad de respuesta ante los requerimientos cambiantes de los distribuidores, además de poder mantener un balance apropiado entre la explotación del conocimiento que ha sido generado y la exploración de nuevas oportunidades de mercado (Tax y Brown, 1998). De aquí se puede derivar que:

H1. El AO del fabricante tiene un efecto positivo en la satisfacción del principal distribuidor con el fabricante.

\subsection{Aprendizaje organizativo y lealtad}

La lealtad en las relaciones comprador-vendedor es uno de los objetivos prioritarios en las estrategias comerciales de las empresas. Los clientes leales es más que probable que busquen beneficios en el largo plazo y que se involucren en acciones cooperativas que favorezcan a los socios comerciales (Agustin y Singh, 2005). La lealtad se considera una fuente de ventaja competitiva cuyas consecuencias se pueden materializar en menores costes en la obtención de nuevos clientes, mayor estabilidad en el crecimiento de las ventas, y poder contar con clientes que actúan como defensores de la empresa (Mithas et al., 2005). La lealtad se define como la intención de llevar a cabo un conjunto variado de comportamientos que ponen de manifiesto la motivación por mantener la relación de intercambio, tales como: la compra repetida, la generación de referencias positivas, y la tolerancia al precio (Jacoby y Chestnut, 1978; Lam et al., 2004).

Ahora bien, al igual que con la satisfacción, el AO se puede relacionar con la lealtad. Cuando el fabricante tiene un alto nivel de AO se encontrará en una posición favorable para identificar y resolver los problemas de su contraparte (Reichheld, 1996). Esto le permitirá llevar a cabo acciones organizativas que fortalecerán la frecuencia y magnitud de los intercambios relacionales. Los programas de lealtad de los fabricantes, por ejemplo, tienen un papel relevante en estas circunstancias, ya que ayudarán a desarrollar y mantener la lealtad del distribuidor, la cual, como se ha comentado con anterioridad, tiene un componente actitudinal y otro de comportamiento. De este modo, parece razonable pensar que el fabricante que actúa como una organización de aprendizaje será capaz de identificar y establecer las recompensas necesarias para que se produzca la compra repetida del distribuidor. El fabricante también será capaz de adoptar aquellas iniciativas con las que mejorar la predisposición del distribuidor para generar referencias positivas y tener una mayor tolerancia al precio de los productos que éste le ofrece (Wallace et al., 2004). Conforme a los argumentos mostrados se propone que:

H2. El AO del fabricante tiene un efecto positivo en la lealtad del principal distribuidor con el fabricante.

\subsection{Satisfacción, lealtad y resultados empresariales}

La satisfacción se ha mostrado a menudo como un importante antecedente de la lealtad (Spiteri y Dion, 2004). Tal es así, que cuando un fabricante consigue satisfacer al distribuidor en una serie de transacciones, éste último es muy probable que quiera continuar con la relación, especialmente si nunca ha existido algún tipo de desavenencia con el 
fabricante. Lo anterior permite plantear la siguiente hipótesis de investigación:

H3. La satisfacción del principal distribuidor con el fabricante tiene un efecto positivo en la lealtad que este distribuidor experimenta con el fabricante.

Existen varias razones por las que la satisfacción puede favorecer los resultados empresariales (Luo y Homburg, 2007). Una es la premisa de que si el fabricante puede obtener satisfacción del distribuidor en las sucesivas interacciones, éste último es probable que quiera mantener la relación, y esto significará que habrá un flujo constante de ingresos para el fabricante en el futuro. También es razonable asumir que, una vez el distribuidor está satisfecho, será más tolerable con los precios fijados por el fabricante. Otro aspecto relevante es que la satisfacción del distribuidor puede reducir los costes de adquirir nuevos socios comerciales como resultado de los comentarios favorables realizados por los distribuidores que han trabajado con el fabricante hasta la fecha (Brown et al., 2005). Finalmente, debido al efecto acumulativo de la satisfacción previa, el distribuidor puede expresar este estado afectivo positivo incrementando su demanda por los productos y servicios, impulsando así los flujos de caja del fabricante y reduciendo los costes de transacción. Este razonamiento nos permite afirmar que:

H4. La satisfacción del principal distribuidor con el fabricante tiene un efecto positivo en los resultados empresariales que experimenta dicho fabricante.

Si el fabricante constata que el distribuidor es leal, actuando como un socio comercial y mostrando un comportamiento de compras repetidas, generación de referencias positivas, y tolerancia al precio, entonces es posible esperar una mejora en los resultados empresariales del fabricante (Spiteri y Dion, 2004). Trabajar con un distribuidor que muestra tal lealtad es un factor clave para que la relación tenga una orientación de largo plazo. Este tipo de distribuidor se puede esperar que compre más a lo largo del tiempo, e incluso que incremente su presupuesto de compra con el fabricante si sus ingresos aumentan. Los distribuidores que son leales están también preparados para emitir opiniones favorables y prestar más atención a los productos del fabricante porque asocian éste último con una propuesta única de valor que excede el valor ofrecido por la competencia. Todo esto favorece la reputación del fabricante en su quehacer diario, y atrae así la atención de nuevos distribuidores sin que ello suponga un incremento en los costes de adquisición de nuevos clientes (Cre- tu y Brodie, 2007). Estos argumentos nos conducen a la siguiente hipótesis de investigación:

H5. La lealtad del principal distribuidor con el fabricante tiene un efecto positivo en los resultados empresariales que experimenta dicho fabricante.

\subsection{Aprendizaje organizativo y resultados empresariales}

El AO de una empresa puede ser uno de los principales precursores de los resultados que ésta alcanza en el mercado donde se desenvuelve (Tippins y Sohi, 2003). En este trabajo los resultados empresariales se identifican como la consecución de los objetivos organizativos con respecto al crecimiento de las ventas, la cuota de mercado, los beneficios, y la rentabilidad (Santos et al., 2005). De acuerdo con el enfoque basado en los recursos (EBR), el aprendizaje del fabricante tiene un efecto positivo en los resultados empresariales (Hult et al., 2002). Precisamente, el $A O$ es un recurso estratégico que permite la consecución de ventajas competitivas de carácter sostenible: primero, porque es valioso al favorecer la creación de ofertas de mercado que son deseadas por los distribuidores; segundo, porque es escaso o raro dado que los estudios empíricos siguen confirmando sus efectos positivos en los resultados empresariales; y tercero, porque es intangible lo que lo convierte en difícil de imitar por los competidores, a la vez que dificulta su transferencia desde otras organizaciones. Todo ello se traduce en la siguiente hipótesis de investigación:

H6. El AO del fabricante tiene un efecto positivo en los resultados empresariales que experimenta dicho fabricante.

\section{Metodología}

\subsection{Muestra y recogida de información}

A partir de la base de datos SABI se establece una población final de 1820 empresas caracterizadas por: (a) ser fabricantes establecidos en España, (b) pertenecer a más de un sector de actividad económica, y (c) disponer de un tamaño mediano conforme al criterio de la Unión Europea (UE) de 2003 (Tabla 1). La persona encuestada en las organizaciones fue el Director General, Gerente o Director Comercial. Antes de remitir el cuestionario, todas y cada una de las empresas de la población fueron contactadas telefónicamente. En todos los casos se comprobó que las 
empresas estuviesen certificadas conforme a la norma ISO 9001:2000. Se garantiza así que las empresas analizadas desarrollan bases regulares de información sobre sus clientes.

Así mismo, se realizó satisfactoriamente un test de competencia del informante clave. El cuestionario estructurado se elaboró después de realizar una amplia labor de revisión de la literatura. Se efectuó adicionalmente un pretest a través de entrevistas en profundidad con cinco académicos de reconocido prestigio y tres altos directivos conocedores del tema objeto de estudio. El trabajo de campo concluyó con la obtención de 181 respuestas válidas, lo que se corresponde con una tasa de respuesta del $9,95 \%$. Esta tasa es similar a la obtenida en otras investigaciones relacionadas con el $\mathrm{AO}$ en el ámbito del marketing (Santos et al., 2005). Por último, al estudiar el sesgo de no-respuesta, se siguió el procedimiento sugerido por Armstrong y Overton (1977). Los resultados sugieren que no existen diferencias significativas entre los primeros en contestar el cuestionario y los que lo hicieron en último lugar (Tabla 9-1).

\subsection{Escalas de medida}

Se han utilizado escalas Likert de siete puntos. Con respecto al AO, éste se considera como un sistema de procesamiento de información que involucra la adquisición, distribución, e interpretación de la información además de la memoria organizativa (Huber, 1991; Moorman, 1995; Sinkula, 1994). Se ha empleado una escala existente para medir dicho factor desarrollada por López et al. (2010). Esta escala distingue dentro de la adquisición de información
(Al) entre: (a) la Al directa, que surge cuando la organización consigue información de su experiencia directa, y (b) la Al indirecta, que centra su atención en la información capturada a través de la experiencia de otras entidades. La distribución de información recoge la trascendencia de una diseminación eficaz de la información en el proceso de aprendizaje. La interpretación de información considera lo útil que resulta para una empresa una comprensión lo más amplia y uniforme posible de la información. Para la última dimensión, la memoria organizativa, se trabajó con aquel tipo de memoria que está basado en los individuos y las redes sociales que éstos generan.

También examinamos el resto de escalas durante el pre-test. Se confirmó que los ítems adaptados eran adecuados para nuestro dominio de investigación. Para medir las variables de resultado relacional (satisfacción y lealtad del distribuidor) se utilizaron versiones modificadas de escalas previamente desarrolladas. La satisfacción del distribuidor más importante percibida por el fabricante (SA) se evaluó como un concepto global, de carácter acumulativo, donde el fabricante valora hasta qué punto el distribuidor estima que la relación de la que forma parte ha superado las expectativas que había puesto en ella. Se utilizó la escala de Cannon y Perreault (1999) adaptada al contexto que nos ocupa.

Con respecto a la lealtad del distribuidor más importante percibida por el fabricante (LE), cabe destacar que se ha medido aquel tipo de lealtad que combina aspectos de comportamiento y actitud. Para la lealtad comportamental se

\section{Tabla 1. Ficha técnica de la investigación}

Población objetivo

Unidad muestral

Ámbito

Método de recogida de información

Censo poblacional

Tamaño muestral

Procedimiento de muestreo
Empresas medianas fabricantes pertenecientes a los sectores de alimentación (CNAE: 15), químico y plástico (24 y 25), siderurgia y metal $(26,27$ y 28$)$, otra maquinaria (29), maquinaria y equipo eléctrico, electrónico y óptico (30, 31, 32 y 33 ) y equipos de transporte (34 y 35 )

Director General, Gerente o Director Comercial

Nacional

Cuestionario estructurado proporcionado a directivos a través del correo electrónico o bien el fax

1820

181

La elección de los sectores fue discrecional, contactando telefónicamente con la totalidad de las 1820 empresas fabricantes 
han utilizado dos ítems, LE1 y LE3, que hacen mención a un acto de compra repetido a lo largo del tiempo. Los indicadores restantes se corresponden con la perspectiva actitudinal de la lealtad. La generación de referencias positivas viene representada por el ítem LE2, mientras que la tolerancia al precio se valora por medio de LE5. Se incluyen también dos indicadores, LE4 y LE6, que capturan la disposición actitudinal positiva, a nivel general, que el distribuidor tiene en proseguir la relación.

Para la escala de los resultados empresariales (RE) se solicita al encuestado que valore una serie de ítems ampliamente utilizados en la literatura, tales como: el crecimiento de las ventas, la cuota de mercado, los beneficios, y la ROI, return on investment (Tippins y Sohi, 2003).

\section{Resultados}

Los resultados empíricos fueron evaluados siguiendo procedimientos y técnicas estadísticas ampliamente reconocidas en la literatura. Primero, las propiedades psicométricas (fiabilidad, validez convergente, y validez discriminante) de las escalas de medida fueron examinadas siguiendo las recomendaciones metodológicas de Churchill (1979), Gerbing y Anderson (1988) y Slater et al. (2010). Segundo, las hipótesis formuladas fueron analizadas por medio de un sistema de ecuaciones estructurales.

\subsection{Modelos de medida}

Para analizar las escalas de medida, se realizaron varios análisis factoriales confirmatorios. El procedimiento de estimación utilizado es el de máxima verosimilitud robusta para evitar los problemas asociados con la no normalidad de los datos (Bentler, 1995). Las escalas de medida fueron divididas en dos subconjuntos de variables: (a) los factores de orden inferior relacionados con la estructura factorial de orden superior del AO, esto es, la adquisición de información directa, la adquisición de información indirecta, la distribución de información, la interpretación de información, y la memoria organizativa; y (b) la satisfacción, la lealtad, y los resultados empresariales. Se utilizó esta metodología para no exceder la recomendación de cinco casos por cada parámetro estimado (Benler \& Cho, 1988). La calidad del ajuste de los modelos de medida fue evaluada utilizando: S-B $X^{2}$ (Satorra-Bentler chi cuadrado), Bentler-Bonnett Non-Normed Fit
Index (NNFI), Comparative Fit Index (CFI), Incremental Fit Index (IFI), Standardized Root Mean Square Residual (SRMR), y Root Mean Square Error of Approximation (RMSEA) (Bentler, 1995).

De forma más precisa, para verificar la naturaleza multi-dimensional del AO se llevaron a cabo análisis factoriales confirmatorios de primer, segundo y tercer orden. El modelo factorial confirmatorio de primer orden (NNFI $=0.96, \mathrm{CFI}=0.97, \mathrm{IFI}=0.97, \mathrm{SRMR}=0.05$, RMSEA = 0.05) (Tabla 2) sugirió el empleo de tres indicadores para medir la adquisición de información directa (Índice de Fiabilidad Compuesta, IFC $=0.85$, Varianza Media Extraída, AVE $=0.67)$, tres indicadores para medir la adquisición de información indirecta (IFC = 0.83 , AVE $=0.61$ ), cuatro indicadores para medir la distribución de información (IFC $=0.88$, AVE $=0.66)$, cuatro indicadores para medir la interpretación de información (IFC $=0.88$, AVE $=0.64)$, y cuatro indicadores para medir la memoria organizativa (IFC $=0.87, \mathrm{AVE}=$ 0.62). En el proceso de purificación, se eliminaron los indicadores que no convergieron adecuadamente en sus respectivas variables latentes (Gerbing y Anderson, 1988). En lo que se refiere al modelo factorial confirmatorio de segundo orden $(\mathrm{NNFI}=0.96, \mathrm{CFI}=0.96$, $\mathrm{IFI}=0.96$, SRMR $=0.05$, RMSEA $=0.05$ ), examinamos si la adquisición de información era un factor de orden superior conformado por la adquisición de información directa e indirecta. En el modelo factorial confirmatorio de tercer orden, se asumió que los factores obtenidos en el modelo factorial de segundo orden, esto es, adquisición de información, distribución de información, interpretación de información, y memoria organizativa emanaban del AO. Los resultados empíricos sugieren que los tres modelos factoriales confirmatorios anteriormente mencionados ajustan bien con los datos (Tabla 2). Además, el modelo factorial confirmatorio de tercer orden mostró unos índices de ajuste más altos y unos criterios de comparación más bajos - el Akaike Information Criterion (AIC) y el Consistent AIC (CAIC) (Steenkamp y Baumgartner, 1998) - que los presentes en los modelos factoriales de primer y segundo orden. En el modelo factorial confirmatorio anteriormente mencionado se obtuvieron unos NNFI (0.96), CFI (o.97), e IFI (0.97) todos ellos por encima de 0.95 , y un SRMR $=0.05$, y un RMSEA $=$ 0.05 . De este modo se mostró que el $A O$ es un factor de orden superior. 
Tabla 2. Resultados del análisis factorial confirmatorio

\begin{tabular}{|c|c|c|}
\hline Escalas de medición & Lambda estandarizado & T-valor robusto \\
\hline \multicolumn{3}{|l|}{ Modelo de primer orden } \\
\hline Adquisición información directa.......Al1 & 0.83 & 14.03 \\
\hline 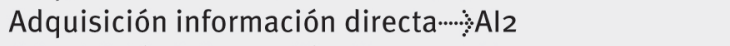 & 0.79 & 9.83 \\
\hline Adquisición información directa......Al3 & 0.81 & 10.57 \\
\hline Adquisición información indirecta......A9 & 0.72 & 9.42 \\
\hline Adquisición información indirecta.....।A10 & 0.84 & $13 \cdot 33$ \\
\hline 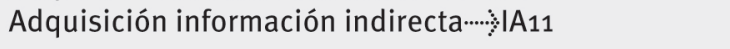 & 0.79 & 10.722 \\
\hline 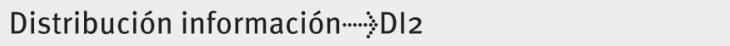 & 0.75 & 10.18 \\
\hline Distribución información....:DI4 & 0.84 & $14 \cdot 35$ \\
\hline Distribución información -.... & 0.84 & 16.58 \\
\hline Distribución información....’. DI6 & 0.81 & 15.41 \\
\hline Interpretación información......ll1 & 0.79 & 12.27 \\
\hline Interpretación información.....।l2 & 0.84 & 14.03 \\
\hline Interpretación información......ll5 & 0.78 & 13.03 \\
\hline 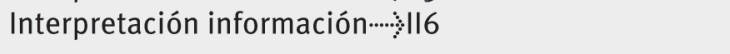 & 0.79 & 14.28 \\
\hline Memoria organizativa $\cdots \cdot \mathrm{OM}_{4}$ & 0.80 & 12.53 \\
\hline Memoria organizativa $\cdots \mathrm{OM}_{5}$ & 0.83 & 11.44 \\
\hline Memoria organizativa $\cdots . \mathrm{OM} 6$ & 0.77 & $9 \cdot 30$ \\
\hline Memoria organizativa $\cdots . . \mathrm{OM}_{7}$ & 0.75 & 11.34 \\
\hline \multicolumn{3}{|l|}{ Modelo de segundo orden } \\
\hline Adquisición información......Al Directa & 0.91 & 10.98 \\
\hline Adquisición información.....Al Indirecta & 0.85 & 8.57 \\
\hline \multicolumn{3}{|l|}{ Modelo de tercer orden } \\
\hline Aprendizaje organizativo -...’Adquisición información & 0.94 & 10.43 \\
\hline Aprendizaje organizativo-..-’’ Pistribución información & 0.93 & 9.49 \\
\hline Aprendizaje organizativo....̀Interpretación información & 0.90 & 10.92 \\
\hline Aprendizaje organizativo $\cdots$ Memoria organizativa & 0.81 & 9.41 \\
\hline \multicolumn{3}{|l|}{ Índices de ajuste } \\
\hline \multicolumn{3}{|c|}{ Modelo de primer orden: S-B X2 $(125)=174.36, \mathrm{NNFI}=0.96, \mathrm{CFI}=0.97, \mathrm{IFI}=0.97$, } \\
\hline \multicolumn{3}{|c|}{ SRMR $=0.05$, RMSEA $=0.05, A I C=-75.64$ CAIC $=-600.45$} \\
\hline \multirow{2}{*}{\multicolumn{3}{|c|}{$\begin{array}{l}\text { Modelo de segundo orden: } \mathrm{S}-\mathrm{B} \mathrm{X}_{2}(128)=181.27, \mathrm{NNFI}=0.96, \mathrm{CFI}=0.96, \mathrm{IFI}=0.96 \text {, } \\
\mathrm{SRMR}=0.05, \mathrm{RMSEA}=0.05, \mathrm{AIC}=-74.74, \mathrm{CAIC}=-612.14\end{array}$}} \\
\hline & & \\
\hline \multicolumn{2}{|c|}{ Modelo de tercer orden: $\mathrm{S}-\mathrm{B} \mathrm{X}_{2}(130)=180.58, \mathrm{NNFI}=0.96, \mathrm{CFI}=0.97, \mid \mathrm{FI}=0.97$} & \\
\hline
\end{tabular}

Con respecto al modelo factorial confirmatorio de primer orden del otro subconjunto de variables, la estructura de medida (Tabla 3) sugirió el uso de cinco indicadores para medir la satisfacción (IFC $=0.96$, AVE $=0.82$ ), el uso de seis indicadores para medir la lealtad (IFC $=0.91$, AVE $=0.62$ ), y el uso de tres indicadores para medir los resultados empresariales (IFC $=0.89$, $A V E=0.74)$. Después de eliminar los indicadores que no convergieron adecuadamente en sus respectivas variables latentes (Gerbing y Anderson,
1988), los índices de ajuste del modelo mostraron unos resultados satisfactorios $(\mathrm{NNFI}=0.96$, $\mathrm{CFI}=0.97, \mathrm{IFI}=0.97, \mathrm{SRMR}=0.05$, y RMSEA $=$ 0.05) (Tabla 3).

En todos los modelos factoriales confirmatorios anteriormente mencionados, la validez convergente se corroboró mediante las estimaciones de los parámetros estandarizados y sus t-valores robustos asociados; todos ellos estando por encima de los umbrales recomendados (Churchill, 1979; Gerbing 
Tabla 3. Resultados del análisis factorial confirmatorio

\begin{tabular}{|c|c|c|}
\hline Escalas de medida & Lambda estandarizado & T-valor robu \\
\hline \multicolumn{3}{|l|}{ Modelo de primer orden } \\
\hline 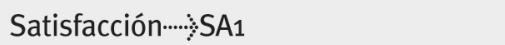 & 0.93 & 12.10 \\
\hline Satisfacción …:SA2 & 0.91 & 13.05 \\
\hline Satisfacción.......SA3 & 0.87 & 11.01 \\
\hline Satisfacción $\cdots . . \mathrm{SA}_{4}$ & 0.94 & 13.03 \\
\hline Satisfacción $\cdots \cdot \mathrm{SA}_{5}$ & 0.88 & 10.85 \\
\hline Lealtad $\cdots \cdot$ LE1 & 0.80 & $14 \cdot 39$ \\
\hline Lealtad $\cdots$ LE2 & 0.74 & 12.10 \\
\hline Lealtad $\cdots$...E3 & 0.82 & 12.88 \\
\hline Lealtad $\cdots . . .54$ & 0.87 & 11.60 \\
\hline Lealtad $\cdots$ LE5 & 0.73 & 11.93 \\
\hline Lealtad $\cdots$...L6 & 0.78 & 10.17 \\
\hline Resultados empresariales-....8RE1 & 0.94 & 13.63 \\
\hline Resultados empresariales..... RE2 & 0.88 & 12.23 \\
\hline Resultados empresariales.....RE3 & 0.74 & 10.35 \\
\hline
\end{tabular}

Fuente: elaboración propia.

y Anderson, 1988; Slater et al., 2010). Como confirmación de la validez discriminante, para cada par de variables latentes la raíz cuadrada de la AVE superó las correlaciones entre variables latentes (Fornell y Larcker, 1981). También se examinó post-hoc la posibilidad de que existiera varianza del método común (a) utilizando el test de un factor único de Harman, y (b) controlando los efectos de un factor de métodos latente no medido - unmeasured latent methods factor approach (Podsakoff et al., 2003). Los resultados obtenidos a partir de las técnicas anteriormente mencionadas revelan que la varianza del método común no es un problema en esta investigación. Por último, todas las escalas de medida resultaron ser fiables, ya que excedieron los estándares para su aceptación.

\subsection{Modelo estructural}

En este apartado se procedió con el análisis de las relaciones causales del modelo estructural especificado: H1-H6, recogidas en la Tabla 4. Antes de nada fue necesario verificar la existencia de validez discriminante. Simplificamos por tanto el factor de orden superior, esto es, el AO, tomando la media de las puntuaciones de cada uno de los factores de orden inferior. Este procedimiento está reconocido en la literatura y se emplea cuando hay un número elevado de ítems y escalas de medida multidimensionales, de tal forma que el número de parámetros a estimar sea el apropiado para el tamaño de la muestra (Hibbard et al., 2001). Los resultados del análisis factorial confirmatorio de primer orden teniendo en cuenta los factores anteriormente mencionados mostró una buena calidad del ajuste. Esto lo corrobora la fiabilidad y la validez convergente de estos factores, así como su validez discriminante.

A la vista de los resultados precedentes, continuamos con el análisis del modelo estructural bajo unas condiciones idóneas. Los resultados muestran que los índices de ajuste son en su conjunto aceptables (Tabla 4). Precisamente, se constató que la influencia del AO del fabricante en la satisfacción del distribuidor era significativa (H1). Sin embargo, se observó un comportamiento diferente en su efecto en la lealtad del distribuidor porque la relación causal no fue significativa (H2). Una posible explicación puede deberse al efecto mediador que ejerce la satisfacción del distribuidor: AO del fabricante..... Satisfacción del distribuidor..... Lealtad del distribuidor (0.30 x 0.79 $=0.24)$. Es decir, el $A O$ del fabricante potencia la satisfacción del distribuidor y ésta, a su vez, potencia la lealtad. El efecto indirecto se estima en 0,24.

La conocida relación entre la satisfacción del distribuidor y la lealtad del distribuidor fue

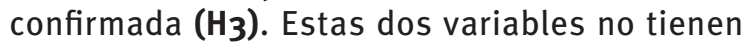
además un impacto significativo en los resultados empresariales, por lo que las hipótesis $\mathrm{H}_{4}$ y $\mathrm{H}_{5}$ fueron rechazadas. Finalmente, se confirmó que el AO del fabricante mejora sus resultados empresariales (H6). 


\section{Tabla 4. Resultados del modelo estructural}

\begin{tabular}{|c|c|c|c|c|}
\hline $\begin{array}{c}\text { Relaciones } \\
\text { causales o } \\
\text { paths del modelo } \\
\text { medida }\end{array}$ & $\begin{array}{c}\text { Signo } \\
\text { Esperado }\end{array}$ & $\begin{array}{c}\text { Coeficiente } \\
\text { Estandarizado }\end{array}$ & $\begin{array}{l}\text { T-Valor } \\
\text { Robusto }\end{array}$ & $\begin{array}{c}\text { Bondad del } \\
\text { Ajuste }\end{array}$ \\
\hline $\mathrm{H}_{1}: \mathrm{AO} \cdots \mathrm{SA}$ & + & 0,30 & 3,02 & $\mathrm{~S}-\mathrm{B} \mathrm{X}_{2}$ \\
\hline $\mathrm{H}_{2}: \mathrm{AO} \cdots$ LE & + & 0,06 & n.s. & $(129)=188,75$ \\
\hline $\mathrm{H}_{3}: \mathrm{SA} \cdots \cdot \mathrm{LE}$ & + & 0,79 & 10,52 & $N N F I=0,95$ \\
\hline $\mathrm{H}_{4}: \mathrm{SA} \cdots \mathrm{RE}$ & + & 0,10 & n.s. & $C F I=0,96$ \\
\hline $\mathrm{H}_{5}: \mathrm{LE} \cdots \mathrm{RE}$ & + & 0,16 & n.s. & $|F|=0,96$ \\
\hline$H 6: A O \cdots$ RE & + & 0,35 & 4,02 & $\begin{array}{l}\text { SRMR }=0,05 \\
\text { RMSEA }=0.05\end{array}$ \\
\hline
\end{tabular}

Clave: $\mathrm{AO}=$ Aprendizaje organizativo fabricante, $\mathrm{SA}=$ Satisfacción distribuidor, $\mathrm{LE}=$ Lealtad distribuidor, $\mathrm{RE}=$ Resultados empresariales, n.s. = no significativo.

\section{Discusión y conclusiones}

Este estudio ha examinado el impacto del AO en la satisfacción y la lealtad. Este es un tema particularmente relevante en los contextos empresariales puesto que ayuda a explicar por qué las relaciones comerciales pueden ser exitosas y duraderas (Lapre y Tsikriktsis, 2006). También se examinó una escala de medida del AO tras el correspondiente análisis de la literatura. Así, partiendo de la premisa de que no se puede gestionar aquello que no se puede medir, la presente investigación aporta en el contexto de las relaciones fabricante-distribuidor un instrumento de medida que puede resultar de utilidad para la gestión empresarial. Se reconoce que estamos dando los primeros pasos en un campo de estudio complejo y difícil, pero que no puede ni debe ser ignorado.

Esta investigación se puede considerar pionera puesto que proporciona evidencia de que el $\mathrm{AO}$ del fabricante es un antecedente directo y positivo de la satisfacción del distribuidor. Sugiere que cuando el $\mathrm{AO}$ está lo suficientemente desarrollado en el fabricante éste puede obtener un conocimiento preciso acerca de las necesidades latentes y expresadas de su socio comercial. Este aspecto es valioso para el fabricante porque le permite desarrollar y mejorar su cartera de productos para satisfacer al distribuidor. Del mismo modo se mostró que el $\mathrm{AO}$ del fabricante está relacionado indirectamente (a través de la satisfacción) y de manera positiva con la lealtad del distribuidor. En este sentido, en investigaciones anteriores el $A O$ ha sido identificado como un factor potencial para conseguir satisfacción y lealtad (Lapre y Tsikriktsis, 2006). Nuestros hallazgos proporcionan soporte empírico para esta idea, e implica que el AO es un factor clave de las variables mencionadas con anterioridad. Del mismo modo se constata que el $\mathrm{AO}$ del fabricante afecta a los resultados empresariales directa y positivamente. Este hallazgo coincide con el estudio de Tippins y Sohi (2003), el cual mostró que el AO puede conducir a un mayor grado de resultados empresariales, ya que a medida que las empresas aprenden de sus clientes y competidores tienen mayores probabilidades de conocer cómo ajustar mejor su oferta de productos a las necesidades del mercado.

Sin embargo, la satisfacción y la lealtad carecen de influencia significativa en los resultados empresariales. Una explicación plausible puede ser que el fabricante al dedicar un esfuerzo excesivo en aprender y mejorar en su quehacer diario en la relación con su distribuidor, buscando el mayor grado de satisfacción y lealtad posible de éste último, puede que minusvalore si tal esfuerzo, en la práctica empresarial, es posible materializarlo en sus propios resultados empresariales. Si no fuera consciente de tal circunstancia el fabricante podría caer en la "trampa" de hacer frente a todas la demandas de su socio comercial sin aceptar, por ejemplo, que existe un punto en el que el coste de ajustarse a las necesidades expresadas por el socio comercial puede que no tenga un impacto significativo en los ingresos que finalmente le reporta (Grewal y Tansuhaj, 2001).

La principal implicación empresarial es que parece razonable que un fabricante invierta y destine recursos a mejorar y desarrollar el AO, ya que esto tendrá un efecto favorable en la relación que mantiene con el distribuidor. La preocupación por el aprendizaje en una empresa dará lugar a una búsqueda e interés constante por conocer en detalle los mercados actuales y potenciales donde podría desplegar su actividad comercial. Esto 
puede mejorar la respuesta de la empresa a las nuevas necesidades del distribuidor y a mantener un adecuado equilibrio entre el uso del conocimiento que ha sido acumulado con el paso del tiempo y la exploración de nuevas oportunidades de mercado. De este modo, cuando una empresa fabricante tiene un alto nivel de $\mathrm{AO}$ será más probable que el distribuidor esté satisfecho, y que el distribuidor lo considere como un socio comercial seguro y fiable con el que se puede mantener una relación con una orientación de largo plazo. De hecho, esto parece razonable porque el fabricante tiene más probabilidades de reconocer los errores cometidos y ser capaz de rectificar cualquier curso de acción cuando sea necesario.

Este trabajo presenta, al igual que otros estudios desarrollados en el campo de las relaciones empresariales, las limitaciones típicas asociadas al uso de la investigación basada en encuesta. Primero, al tratarse de un trabajo de carácter transversal se debe tener especial cuidado al hacer inferencias acerca de la evolución futura de las relaciones de causalidad identificadas. Segundo, se ha utilizado un único informante clave por organización. Si bien la varianza del método común no ha sido un problema en este estudio, el empleo de más de un informante clave por organización podría aumentar la fiabilidad de las medidas. Por último, los datos recogidos se obtienen a partir de una muestra de empresas fabricantes españolas que operan en sectores industriales específicos. Este hecho, limita la posibilidad de generalizar inmediatamente los resultados empíricos obtenidos a otro tipo de empresas y requiere la realización de futuros estudios que comprueben las relaciones planteadas en contextos competitivos diferentes al mercado español.

La investigación futura a partir de este estudio también debe incluir otra serie de aspectos. Así, tal y como se ha indicado previamente, el aprendizaje individual es la base del AO. Por tanto, en el substrato del AO está la calidad y cantidad del aprendizaje humano. Sin embargo, el aprendizaje de los individuos está fuertemente condicionado por el entorno en el que se desenvuelven: "el aprendizaje no es sólo un proceso psicológico, está íntimamente ligado al mundo y es afectado por este... las personas adoptan el conocimiento, los valores, creencias y actitudes de la sociedad en la que viven" (Jarvis, 1987, p. 11). En este sentido, Illeris et al. (2004) explican que el aprendizaje depende de tres elementos fundamentales: las características subjetivas de los individuos (elementos biológicos y psicológicos), el entorno socio-cultural y el entorno técnico-administrativo en el que se desenvuelven. Todo ello hace necesario llevar a cabo más investigaciones que teniendo en cuenta estas líneas de investigación, e incorporando la teoría del aprendizaje humano, analicen aspectos como por ejemplo el nivel de AO que se puede llevar a cabo en organizaciones con diferentes tipos de estructuras organizativas o con diferentes culturas empresariales (Jarvis, 2006). Así mismo, entendemos que otros aspectos importantes que merecen la futura consideración de los investigadores son el estudio de los efectos del AO sobre el tipo de estrategias competitivas de las organizaciones, sobre su capacidad de desarrollo de innovaciones exitosas o si existen factores que moderan la conexión causal entre el $\mathrm{AO}$ y los resultados empresariales.

\section{Referencias}

Agustin, C., \& Singh, J. (2005). Curvilinear effects of consumer loyalty determinants in relational exchanges. Journal of Marketing Research, 42 (1), 96-108.

Akgün, A. E., Lynn, G. S., \& Yilmaz, C. (2006). Learning process in new product development teams and effects on product success. A socio cognitive perspective. Industrial Marketing Management, 35 (2), 210-224.

Anderson, J., \& Narus, J. (1984). A model of the distributor's perspective of distributor-manufacturer working relationships. Journal of Marketing, $48(4), 62-74$.

Argyris, C. (1991). Teaching smart people how to learn. Harvard Business Review, 69 (3), pp. 99109.

Argyris, C. (1996). Actionable knowledge: Design casuality in service of consequential theory. Journal of Applied Behavioral Science, 32 (4), 390-406.

Argyris, C., \& Schön D (1978). Organizational learning: $a$ theory in action perspective. New York: Addison-Wesley.

Argyris, C., \& Schon, D.A. (1996). Organizational learning II. Theory, method and practice. Massachusetts: Addison-Wesley.

Armstrong, J. S., \& Overton, T. S. (1977). Estimating no response bias in mail surveys. Journal of Marketing Research, 14 (3), 396-402. 
Bell, S., Menguc, B., \& Widing, R. E. (2010). Salesperson learning, organizational learning, and retail store performance. Journal of the Academy of Marketing Science, 38 (2), 187-201.

Bell, S., Whitwell, G., \& Lukas, B. (2002). Schools of thought in organizational learning. Journal of the Academy of Marketing Science, 30 (1), 70-86.

Bentler, P. M. (1995). EQS: Structural equations program manual. BMDP Statistical Software, Los Angeles, California.

Bentler, P.M., \& Cho. C.P. (1998). Practical Issues in Structural Modeling, in Common Problems/ Proper Solutions: Avoiding Error in Quantitative Research, Long, J. S. (Ed.), Sage Publications, Newbury Park, CA, 161-192.

Bontis, N., Crossan, M. M., \& Hulland, J. (2002). Managing an organizational learning system by aligning stocks and flows. Journal of Management Studies, 39 (4), 437-469.

Brown, T., Barry, T., Dacin, P. A., \& Gunst, R. (2005). Spreading the word. Journal of the Academy of Marketing Science, 33 (2), 123-138.

Cannon, J., \& Perreault, W. (1999). Buyer-seller relationships in business markets. Journal of Marketing Research, 36 (4), 439-460.

Churchill, G. A. (1979): “A paradigm for developing better measures of marketing constructs". Journal of Marketing Research, 16 (1), 64-73.

Cretu, A., \& Brodie, R. (2007). The influence of brand image and company reputation where manufacturers market to small firms. Industrial Marketing Management, 36 (2), 230-240.

Cross, R., \& Baird, Ll. (2000). Technology is not enough: Improving performance by building organizational memory. Sloan Management Review, 41 (3), 69-78.

Cross, R., Liedtka, J., \& Weiss, L. (2005). A practical guide to social networks. Harvard Business Review, 83 (3), 124-132.

Edmonson, A. (2008). The competitive imperative of learning. Harvard Business Review. 86 (7/8), 60-67.

Fornell, C., \& Larcker, D. F. (1981). Evaluating structural equation models with unobservable variables and measurement error. Journal of Marketing Research, 18 (1), 39-50.

Ganesan, S. (1994). Determinants of long-term orientation in buyer-seller relationships. Journal of Marketing, 58 (2), 1-19.

Gerbing, D., \& Anderson, J. (1988). An updated paradigm for scale development incorporating unidimensionality and its assessment. Journal of Marketing Research, 25 (2), 186-192.

Grewal, R., \& Tansuhaj, P. (2001). Building organizational capabilities for managing economic crisis. Journal of Marketing, 65 (2), 67-80.

Hibbard, J. D., Kumar, N., \& Stern, L. W. (2001). Examining the impact of destructive acts in marketing channel relationships. Journal of Marketing Research, 38 (1), 45-61.

Huber, G. (1991). Organizational learning, Organization Science, 2 (1), 88-115.

Hult, T. (1998). Managing the international strategic sourcing process as a market driven organizational learning system. Decision Sciences, 29 (1), 193-216.

Hult, T., Ketchen, D., \& Slater, S. (2002). A longitudinal study of the learning climate and cycle time in supply chains. Journal of Business \& Industrial Marketing, 17 (4), 302-323.

Illeris, K., \& Associates (2004). Learning in working life. Copenhagen: Roskilde University Press.

Ittner, C., \& Larcker, D. (2003). Coming up short on nonfinancial performance measurement. Harvard Business Review, 81 (11), 88-95.

Jacoby, J., \& Chestnut, R. (1978). Brand loyalty measurement and management. New York: John Wiley \& Sons.

Jarvis, P. (1987). Adult education in the social context. London: Croom Helm.

Jarvis, P. (2006). Towards a comprehensive theory of adult learning. London: Routledge.

Lam, S., Shankar, V., Erramilli, K., \& Murthy, B. (2004). Customer value, satisfaction, loyalty, and switching costs. Journal of the Academy of Marketing Science, 32 (3), 293-311. 
Lapre, M., \& Tsikriktsis, N. (2006). Organizational learning curves for customer dissatisfaction. Management Science, 52 (3), 352-366.

López, J. A., Santos, M. L., \& Trespalacios, J. (2010). Organisational learning and value creation in business markets. European Journal of Marketing, 44 (11/12), 1612-1641.

Luo, X., \& Homburg, C. (2007). Neglected outcomes of customer satisfaction. Journal of Marketing, 71 (2), 133-149.

Mithas, S., Krishnan, M.S., \& Fornell, C. (2005). Why do customer relationship management applications affect customer satisfaction. Journal of Marketing, 69 (4), 201-209.

Moorman, C. (1995). Organizational Market Information Processes: Cultural Antecedents and New Product Outcomes. Journal of Marketing Research, 32 (3), 318-335.

Morgan, R., \& Turnell, C. (2003). Market based organizational learning and market performance gains. British Journal of Management, 14 (3), 255-274.

Narayandas, D. (2005). Building loyalty in business markets. Harvard Business Review, 83 (9), 1-9

Palmatier, R. W., Dant R. P., \& Grewal, D. (2007). A comparative longitudinal analysis of theoretical perspectives of interorganizational relationship performance. Journal of Marketing, 71 (4), 179194.

Podsakoff, P. M., Mackenzie, S. B., Lee, J. \& Podsakoff, N. P. (2003). Common method biases in behavioral research: A critical review of the literature and recommended remedies, Journal of Applied Psychology, 88 (5), 879-903.

Reichheld, F. (1996). Learning from defections. Harvard Business Review, 74 (2), 56-69.

Santos, M.L., Sanzo, M., Álvarez, L., \& Vázquez, R. (2005). Organizational learning and market orientation. Industrial Marketing Management, 34 (3), 187-202.

Schein, E. H. (1993). How can organizations learn faster? The challenge of entering the green room. Sloan Management Review, 34 (2), 85-92.
Sinkula, J. M. (1994). Market information processing and organizational learning. Journal of Marketing, 58 (1), 35-45.

Slater, S. F. \& Narver, J. C. (1995). Market orientation and the learning organization. Journal of Marketing, 59 (3), 63-74.

Slater, S., Hult, T., \& Olson, E. (2010). Factors influencing the relative importance of marketing strategy creativity and marketing strategy implementation effectiveness. Industrial Marketing Management, 39 (4), 551-559.

Spiteri, J., \& Dion, P. (2004). Customer value, overall satisfaction, end user loyalty, and market performance. Industrial Marketing Management, 33 (8), 675-687.

Steenkamp, J. E. M., \& Baumgartner, H. (1998). Assessing measurement invariance in crossnational consumer research, Journal of Consumer Research, 25 (1), 78-90.

Tax, S., \& Brown, S. (1998). Recovering and learning from service failure. Sloan Management Review, 40 (1), $75-88$.

Tippins, M., \& Sohi, R. (2003). IT competency and firm performance. Strategic Management Journal, 4 (8), 745-761.

Treviño, L.K., Webster, J., \& Stein, E. W. (2000). Making connections: Complementary influences on communication choices, attitudes and use. Organization Science, 11 (2), 163-182.

Tucker, A. L., Nembhard, I. M., \& Edmonson, A. C. (2007). Implementing new practices: An empirical study of organizational learning in hospital intensive care units. Management Science, 53 (6), 894-907.

Wallace, D., Giese, J., \& Johnson, J. (2004). Customer retailer loyalty in the context of multiple channel strategies. Journal of Retailing, 80 (4), 249-263.

Wernerfelt, B. (1984). A resource based view of the firm. Strategic Management Journal, 5 (2), 171180. 